H. IMAI

KODAI MATH. J.

3 (1980), 56-58

\title{
ON THE RATIONAL POINTS OF SOME JACOBIAN VARIETIES OVER LARGE ALGEBRAIC NUMBER FIELDS
}

\author{
By HidEO IMAI
}

In this note we shall prove the following: Let $X$ be a hyperelliptic curve defined over the rational number field $\boldsymbol{Q}$ and let $J$ be its Jacobian variety. Let $L$ be the field generated by all square roots of rational integers over $\boldsymbol{Q}$. Then the group of $L$-rational points $J(L)$ has an infinite rank over the rational integer ring $\boldsymbol{Z}$.

In Frey and Jarden [1], the following is conjectured: Let $A$ be an abelian variety defined over $\boldsymbol{Q}$ and $\boldsymbol{Q}_{a b}$ the maximal abelian extension of $\boldsymbol{Q}$. Then does the group $A\left(\boldsymbol{Q}_{a b}\right)$ have an infinite rank over $\boldsymbol{Z}$ ? Our result supports this conjecture partially.

1. Let $X$ be a hyperelliptic curve defined by the equation (in the affine form) $y^{2}=f(x)$, where $f(x)$ is a monic separable polynomial of degree $2 g+1$ with coefficients in $Z$. Let $P_{0}=(\infty, \infty)$ be the point at infinity on $X$, which is rational over $\boldsymbol{Q}$. Let $z=x^{g} / y$ be a local uniformizing parameter at $P_{0}$. Let $\omega_{i}=x^{2-1} d x / y \quad(i=1,2, \cdots, g)$ be the canonical base of the space of differential forms of the first kind on $X$. Writing these $\omega_{i}$ in terms of $z$ and integrating $\omega_{i}$ formally, we get power series $\Psi_{i}(z) \in \boldsymbol{Q}[[z]]$ such that $\Psi_{i}(0)=(0)$ and $\omega_{i}=d \Psi_{\imath}$.

LEMMA 1.

$$
\Psi_{i}(z)=\frac{-2}{2 g-2 \imath+1} z^{2 g-2 \imath+1}+\sum_{n>b-\imath} \frac{c_{n}^{(i)}}{2 n+1} z^{2 n+1} \quad \text { with } \quad c_{n}^{(i)} \in \boldsymbol{Z} .
$$

Proof. It is easily proved by direct computation. We outline the proof. Differentiating $z=x^{g} / y$ with respect to $x$, we have

Hence we have

$$
d z=\left(g x^{g-1}-x^{g} f^{\prime}(x) / 2 f(x)\right) d x / y .
$$

$$
\Psi_{i}^{\prime}(z)=1 / g x^{g-\imath}\left(1-x f^{\prime}(x) / 2 g f(x)\right) .
$$

We write $z=x^{g} / \sqrt{f(x)}$ and expand the above equation in terms of $t=1 / x$. Let $\Psi_{i}(z)=\sum_{n=1}^{\infty} a_{n} z^{n}$ and let $h(1 / x)=f(x) / x^{2 g+1}-1$. After some computations we get

Received January 17, 1979 


$$
-2 t^{g-\imath} \sum_{n=0}^{\infty}\left(t h^{\prime}(t) /(1+h(t))\right)^{n}=\sum_{n=1}^{\infty} n a_{n}(t /(1+h(t)))^{(n-1) / 2} .
$$

Our assertion follows from this directly.

Put $\Psi(z)={ }^{t}\left(\Psi_{1}(z), \cdots, \Psi_{g}(z)\right)$ a $g$-dimensional column vector.

2. Now let $J=\operatorname{Jac}(X)$ be the Jacobian variety of $X$. Choose an imbedding $\Lambda: X \rightarrow J$ defined over $\boldsymbol{Q}$ such that $\Lambda\left(P_{0}\right)=0$ the identity point of $J$. Let $y_{1}, \cdots, y_{g}$ be rational functions on $J$ defined over $\boldsymbol{Q}$ such that they constitute a system of local uniformizing parameters at 0 . Let $\eta_{1}, \cdots, \eta_{g}$ be invariant differential forms on $J$ defined over $\boldsymbol{Q}$ such that $\omega_{i}=\eta_{i} \circ \Lambda(i=1,2, \cdots, g)$. It is well known that these $\eta_{1}, \cdots, \eta_{g}$ form a base of the space of invariant differential forms on $J$. As $\eta_{2}$ is closed (cf. [2], Proposition 1.3 and Lemma 1.4), there exists a formal power series $F_{i}\left(y_{1}, \cdots, y_{g}\right) \in \boldsymbol{Q}\left[\left[y_{1}, \cdots, y_{g}\right]\right]$ such that $F_{i}(0, \cdots, 0)=0$ and $\eta_{\imath}=d F_{2}$. Let $F={ }^{t}\left(F_{1}, \cdots, F_{g}\right)$ and let $\hat{J}$ the formal group of $J$. From [2], Proposition 1.1 and Theorem 1, there is a matrix $A \in G L_{g}(\boldsymbol{Q})$ such that $A F(y) \equiv y(\bmod \operatorname{deg} 2)$ and $A F: \hat{J} \rightarrow \hat{\boldsymbol{G}}_{a}^{g}$ is a strong isomorphism over $\boldsymbol{Q}$ where $\hat{\boldsymbol{G}}_{a}$ is the formal group of the additive group. From [2], Proposition 1.1, we see that each component of the differential $d(A F)$ is obtained from differentiating the formal group law of $\hat{J}$. Hence for a prime $p$ at which $J$ and $y_{\imath}, \eta_{\imath}(i=1, \cdots, g)$ have good reduction, the coefficients of $d(A F)$ are $p$-adic integers. Hence if we write the $i$-th coordinate of $A F$ as $\sum_{n_{1}, \cdots, n_{g}} a_{n_{1}, \cdots, n_{g}}^{(i)} y_{1}^{n_{1}} \cdots y_{g}{ }^{{ }^{g} g}$, we shall have $v_{p}\left(a_{n_{1}, \cdots, n_{\boldsymbol{g}}}{ }^{(i)}\right) \geqq-\min _{1 \leq j \leq g} v_{p}\left(n_{j}\right)$ were $v_{p}$ is the $p$-adic additive valuation. From this we see that $A F$ is convergent in sufficiently small neighbourhood of 0 in the $p$-adic topology. The inverse function theorem (cf. [3], LG 2.10) implies the following:

LEMMA 2. Let $p$ be a prime at which $J$ and $y_{\imath}, \eta_{\imath}(\imath=1, \cdots, g)$ have good reduction, then $(A F)^{-1}$ is convergent in sufficiently small neighbourhood of 0 in the p-adic topology.

3. From the equation $\omega_{i}=d F_{\imath} \circ \Lambda=d \Psi_{\imath}$, we have $\Psi_{\imath}=F_{\imath} \circ \Lambda$ i. e., $\Psi=F \circ \Lambda$. We take a prime $p$ at which $J$ and $y_{\imath}, \eta_{\imath}$ have good reduction. Let $K / \boldsymbol{Q}_{p}$ be a finite extension, $P$ be a $K$-rational point of $X$ and let $m$ be an integer. As $A F: \hat{J} \rightarrow \hat{G}_{a}^{g}$ is an isomorphism, $m \Lambda(P) \in J(K)$ may be computed as $m \Lambda(P)=$ $(A F)^{-1}(m A \Psi(P))$ when the right hand side converges.

Especially we consider the point $P=\left(1 / p^{\alpha}, \sqrt{f\left(1 / p^{\alpha}\right)}\right)$ where $p$ is a prime with the above good reduction condition and $\alpha$ is a sufficiently large odd integer. Let $c=p^{(2 g+1) \alpha} f\left(1 / p^{\alpha}\right)$, then $c \in Z$ and $c$ is coprime to $p$. Let $K=$ $\boldsymbol{Q}(\sqrt{c / p})$, then $P$ is rational over $K$. As $p$ is ramified in $K$, we write $p=\mathfrak{p}^{2}$ and let $K_{\mathfrak{p}}$ be the $\mathfrak{p}$-adic completion of $K$. We consider the point $P$ as a $K_{p}$ rational point. For the local parameter $z=x^{g} / y$, the value of $z$ at $P$ is given by $z_{p}=\sqrt{p^{\alpha} / c}$. From Lemma $1, \Psi(P)=\Psi\left(z_{p}\right)$ is convergent in the $\mathfrak{p}$-adic 
topology. From Lemma 2, $(A F)^{-1}\left(m A \Psi\left(z_{p}\right)\right)$ also coverges for sufficiently large $\alpha$.

LEMMA 3. For almost all primes $p$, if an odd integer $\alpha$ is taken sufficiently large, $m \Lambda(P)$ is not $\boldsymbol{Q}$-rational for any non-zero integer $m$ where $\left.P=\left(1 / p^{\alpha}, \sqrt{f\left(1 / p^{\alpha}\right.}\right)\right)$.

Proof. We exclude the prime $p=2$, the primes at which $J, y_{2}, \eta_{2}$ have bad reduction and the primes $p$ such that there exists a non $p$-unit $a_{\imath \jmath}$ for the matrix $A=\left(a_{\imath j}\right)$. For a prime $p$, take an odd integer $\alpha$ sufficiently large so that in the expansion $\Psi\left(z_{p}\right)$, the term $-2 z_{p}$ has smaller $\mathfrak{p}$-adic valuation than any other terms $(-2 z$ is the smallest degree term in the expansions of the coordinates of $\Psi(z))$. We take $\alpha$ more large if necessary, so that the last coordinate of $(A F)^{-1}\left(\Psi\left(z_{p}\right)\right)$ has $\mathfrak{p}$-adic valuation $v_{p}\left(z_{p}\right)$ (note that $\left.A F(y) \equiv y \bmod \operatorname{deg} 2\right)$. Suppose $m \Lambda(P)=Q$ was a $Q$-rational point of $J$. Then the value $Q_{\imath}$ of $y_{\imath}$ at $Q$ is a rational number. Hence $v_{p}\left(Q_{\imath}\right)$ is an even integer (for the $\mathfrak{p}$-adic valuation, $\left.v_{\mathfrak{p}}(p)=2\right)$. On the other hand it can be seen easily from what the above said, that some coordinate of $m \Lambda(P)=(A F)^{-1}(m A \Psi(P))$ has $\mathfrak{p}$-adic valuation $v_{\mathfrak{p}}\left(z_{p}\right)+$ $v_{\mathfrak{p}}(m)$ which is an odd integer since $v_{\mathrm{p}}\left(z_{p}\right)=\alpha$ is odd. This is a contradiction.

THEOREM. Let $L=\boldsymbol{Q}(\sqrt{d} \mid d \in \boldsymbol{Z})$. Then the group of L-rational points $J(L)$ has an infinite rank over $\boldsymbol{Z}$.

Proof. The proof is entirely similar to that of [1], Theorem 2.2. We include it for the convenience of reader. For a prime number $p_{i}$, put $c_{2}=$ $p_{i}{ }^{(2 g+1) \alpha_{\imath}} f\left(1 / P_{\imath}^{\alpha}{ }\right)$ as before, with $\alpha_{\imath}$ a sufficiently large odd integer. We take an infinite sequence of primes $\left\{p_{n}\right\}_{n=1}^{\infty}$ such that $J$ and $y_{2}, \eta_{2}$ have good reduction at $p_{n}$, and that $\boldsymbol{Q}\left(\sqrt{c_{1} / p_{1}}, \cdots, \sqrt{c_{n} / p_{n}}\right) \cap \boldsymbol{Q}\left(\sqrt{c_{n+1} / p_{n+1}}\right)=\boldsymbol{Q}$ for all $n$. For example, take inductively a prime $p_{n+1}$ unramified in $\boldsymbol{Q}\left(\sqrt{c_{1} / p_{1}}, \cdots, \sqrt{c_{n} / p_{n}}\right) / \boldsymbol{Q}$ with the above good reduction condition. Put $P_{\imath}=\left(1 / p_{\imath}^{\alpha} \imath, \sqrt{f\left(1 / P_{\imath}^{\alpha} i\right)}\right)$, then we claim that $\left\{\Lambda\left(P_{\imath}\right)\right\}_{\imath=1}^{\infty}$ are linearly independent over $Z$. Suppose there was a relation $m_{1} \Lambda\left(P_{1}\right)+\cdots+m_{n} \Lambda\left(P_{n}\right)=0$ with $m_{n} \neq 0$. Write this as $m_{1} \Lambda\left(P_{1}\right)+\cdots+$ $m_{n-1} \Lambda\left(P_{n-1}\right)=-m_{n} \Lambda\left(P_{n}\right)$. The left hand side is $\boldsymbol{Q}\left(\sqrt{c_{1} / p_{1}}, \cdots, \sqrt{c_{n-1} / p_{n-1}}\right)$ rational and the right hand side is $\boldsymbol{Q}\left(\sqrt{c_{n} / p_{n}}\right)$-rational. Hence $m_{n} \Lambda\left(P_{n}\right)$ must be a $\boldsymbol{Q}$-rational point. This contradicts to Lemma 3.

\section{REFERENCES}

[1] G. FREY AND M. JARDEN, Approximation theory and the rank of abelian varieties over large algebraic number fields, Proc. London Math. Soc. 28 (1974), 112-128.

[2] T. Honda, On the theory of commutative formal groups, J. Math. Soc. Japan, 22 (1970), 213-246.

[3] J-P. Serre, Lie algebras and Lie group, Benjamın Inc. New York, (1965).

Department of Mathematics

College of general Education

TOHOKU UNIVERSITY

Kawauchi, Sendai, Japan. 